ANNALES

POLONICI MATHEMATICI

$83.2(2004)$

\title{
An existence and localization theorem for the solutions of a Dirichlet problem
}

\author{
by Giovanni Anello and Giuseppe Cordaro (Messina)
}

\begin{abstract}
We establish an existence theorem for a Dirichlet problem with homogeneous boundary conditions by using a general variational principle of Ricceri.
\end{abstract}

1. Introduction and statement of the result. The aim of this paper is to establish the following result:

THEOREM 1. Suppose that $\Omega$ is a bounded open set in $\mathbb{R}^{N}, N \geq 3$, with sufficiently regular boundary $\partial \Omega$ and $f: \Omega \times \mathbb{R} \rightarrow \mathbb{R}$ is a Carathéodory function. Let $s=2 N /(N-2)$ and suppose that there exist $\gamma \in] 2 / s, 1[$, $p \in] 1, \gamma s-1\left[, \alpha \in L^{\gamma s /(\gamma s-1)}(\Omega)\right.$ and $\beta \in L^{1 /(1-\gamma)}(\Omega)$ such that

$$
|f(x, t)| \leq \alpha(x)+\beta(x)|t|^{p} \quad \text { almost everywhere in } \Omega \times \mathbb{R}
$$

and

$$
\|\alpha\|_{L^{s \gamma /(s \gamma-1)(\Omega)}}^{p-1}\|\beta\|_{L^{1 /(1-\gamma)}(\Omega)}<\frac{(p-1)^{p-1}}{p^{p} c_{1}(\gamma)^{p-1} c_{2}(\gamma)^{p+1}},
$$

where $c_{1}(\gamma)$ and $c_{2}(\gamma)$ are the embedding constants of $W^{1,2}(\Omega)$ in $L^{s \gamma}(\Omega)$ and $L^{(p+1) / \gamma}(\Omega)$ respectively. Then the problem

$$
\begin{cases}-\Delta u=f(x, u) & \text { in } \Omega, \\ u=0 & \text { on } \partial \Omega,\end{cases}
$$

has a strong solution $\bar{u}$ such that $\|\bar{u}\| \leq k$, where $k$ is the smallest real root of the equation

$$
c_{2}(\gamma)^{p+1}\|\beta\|_{L^{1 /(1-\gamma)}(\Omega)} r^{p}+c_{1}(\gamma)\|\alpha\|_{L^{s \gamma /(s \gamma-1)}(\Omega)}-r=0 .
$$

We recall that a strong solution of problem $(\mathrm{P})$ is a function $u \in W_{0}^{1,2}(\Omega) \cap W^{2,2}(\Omega)$ such that

$$
-\Delta u(x)=f(x, u(x)) \quad \text { almost everywhere in } \Omega,
$$

2000 Mathematics Subject Classification: Primary 35J20.

Key words and phrases: Dirichlet problem, Ricceri variational principle. 
while a weak solution of problem (P) is a function $u \in W_{0}^{1,2}(\Omega)$ such that

$$
\int_{\Omega} \nabla u(x) \nabla v(x) d x-\int_{\Omega} f(x, u(x)) v(x) d x=0
$$

for all $v \in W_{0}^{1,2}(\Omega)$.

The proof of Theorem 1 is essentially based on the following general variational principle of $\mathrm{B}$. Ricceri which has already been used in several works $[6,7,8]$.

Theorem A ([5, Theorem 2.5]). Let $X$ a real reflexive Banach space, and $\Phi, \Psi: X \rightarrow \mathbb{R}$ two sequentially weakly lower semicontinuous and Gateaux differentiable functionals. Moreover, assume that $\Psi$ is (strongly) continuous and satisfies

$$
\lim _{\|x\| \rightarrow \infty} \Psi(x)=\infty
$$

For every $r>\inf _{X} \Psi$, put

$$
\varphi(r)=\inf _{x \in \Psi^{-1}(]-\infty, r[)} \frac{\Phi(x)-\inf _{\operatorname{cl}_{w}\left(\Psi^{-1}(]-\infty, r[)\right)} \Phi}{r-\Psi(x)}
$$

where $\mathrm{cl}_{w}\left(\Psi^{-1}(]-\infty, r[)\right)$ is the closure of $\Psi^{-1}(]-\infty, r[)$ with respect to the weak topology. Then, for every $r>\inf _{X} \Psi$ and every $\lambda>\varphi(r)$, the functional $\Phi+\lambda \Psi$ has at least one critical point in $\Psi^{-1}(]-\infty, r[)$.

From Theorem A, we deduce that if there exists $r>\inf _{X} \Psi$ such that

$$
\inf _{x \in \Psi^{-1}(]-\infty, r[)} \frac{\Phi(x)-\inf _{\mathrm{cl}_{w}\left(\Psi^{-1}(]-\infty, r[)\right)} \Phi}{r-\Psi(x)}<\frac{1}{2},
$$

then the functional $\Phi+\frac{1}{2} \Psi$ has at least one critical point in $\Psi^{-1}(]-\infty, r[)$.

We apply Theorem A to suitable functionals $\Phi$ and $\Psi$ satisfying condition (3) and defined on the Sobolev space $W_{0}^{1,2}(\Omega)$ equipped with the norm $\|\cdot\|=\left(\int_{\Omega}|\nabla(\cdot)|^{2} d x\right)^{1 / 2}$.

There are many works in which problem $(\mathrm{P})$ is studied. Nevertheless, because of the type of our assumptions, only a few results can be compared with ours. Among these, we find interesting Theorem 3.1 of [4]. In that result the strong solution of problem $(\mathrm{P})$ belongs to $W_{0}^{1, \sigma}(\Omega) \cap W^{2, \sigma}(\Omega)$ for some $\sigma>N / 2$ and the right hand side is a function $f: \Omega \times \mathbb{R} \rightarrow \mathbb{R}$ which can be discontinuous in both variables while, in our case, $f$ must be a Carathéodory function. In [4], the following condition on $f$ is imposed:

There exist $r>0$ and $\sigma>N / 2$ such that $x \mapsto \sup _{|t| \leq B r}|f(x, t)|$ belongs to $L^{\sigma}(\Omega)$, and its norm in this space is not greater than $r$, where

$$
B=\sup _{u \in W_{0}^{1, \sigma}(\Omega) \cap W^{2, \sigma}(\Omega)} \frac{\operatorname{ess~sup}_{x \in \Omega}|u(x)|}{\|u\|_{L^{\sigma}(\Omega)}} .
$$


It is easily seen that condition (4) is satisfied if $f$ is a Carathéodory function and there exist $\alpha, \beta \in L^{\sigma}(\Omega)$ and $p>1$ such that

$$
|f(x, t)| \leq \alpha(x)+\beta(x)|t|^{p} \quad \text { almost everywhere in } \Omega \times \mathbb{R}
$$

and

$$
\|\alpha\|_{L^{\sigma}(\Omega)}^{p-1}\|\beta\|_{L^{\sigma}(\Omega)}<\frac{(p-1)^{p-1}}{p^{p} B^{p}} .
$$

Conditions (5) and (6) are similar to (1) and (2) respectively but, in our case, the summability conditions on $\alpha$ and $\beta$ are different and this allows us to treat some problems which are not covered by Theorem 3.1 of [4]. A simple example in which conditions (1) and (2) are fulfilled and condition (4) is violated, is the following.

EXAmPle 1 . Let $N=3$ and $\sigma=2$. Then

$$
\tilde{c}:=c_{1}(\gamma)^{p-1} c_{2}(\gamma)^{p+1} \leq m(\Omega)^{\frac{p-1-2 \gamma p+6 \gamma^{2}}{6 \gamma}} c^{2 p},
$$

where

$$
c=\sup _{u \in W_{0}^{1,2}(\Omega)} \frac{\|u\|_{L^{6}(\Omega)}}{\|u\|} .
$$

The exact value of $c^{2}$ is $\frac{2}{3 \pi} \sqrt[3]{\frac{2}{\pi}}$ (see $[3$, p. 115]).

Now, we take two functions $\alpha \in L^{4 / 3}(\Omega), \beta \in L^{3}(\Omega)$ non-negative almost everywhere in $\Omega$ and such that

$$
\|\alpha\|_{L^{4 / 3}(\Omega)}\|\beta\|_{L^{3}(\Omega)}<\frac{9 \pi^{2} \sqrt[3]{\pi^{2}}}{m(\Omega)^{1 / 4} 16 \sqrt[3]{4}} .
$$

Put

$$
f(x, t)=\alpha(x)+\beta(x)|t|^{2} \quad \text { for all }(x, t) \in \Omega \times \mathbb{R} .
$$

Since $s=6$, if we choose $\gamma=2 / 3$ then $1 /(1-\gamma)=3$ and $s \gamma /(s \gamma-1)=4 / 3$. Further, in this setting, we have

$$
\tilde{c} \leq m(\Omega)^{1 / 4} c^{2} .
$$

Thus $\alpha, \beta, f$ satisfy all the assumptions of Theorem 1 with $p=2$ and $\gamma=$ $2 / 3$.

Observe that if $\alpha \notin L^{3 / 2}(\Omega)$, then condition (4) is violated because $\sup _{|t| \leq B r} f(\cdot, t) \notin L^{\sigma}(\Omega)$ for all $\sigma>3 / 2$ and $r>0$.

Another result where the growth condition on $f$ is similar to ours is Theorem 1 of [10]. Nevertheless, if the function $f$ is as in the previous example with $\alpha \notin L^{\infty}(\Omega)$ and $\beta$ is not identically 0 , then $f$ does not satisfy the assumptions in [10]. 
2. Proof of Theorem 1. We put

$$
\Psi(u)=\|u\|^{2}, \quad \Phi(u)=\int_{\Omega}\left(\int_{0}^{u(x)} f(x, t) d t\right) d x
$$

for all $u \in W_{0}^{1,2}(\Omega)$. Thanks to condition (2) the operator $\Phi$ is well defined, Gateaux differentiable and sequentially weakly lower semicontinuous. So is the operator $\Psi$, which is strongly continuous as well. Now, the weak solutions of problem $(\mathrm{P})$ are exactly the critical points of the functional $\frac{1}{2} \Psi+\Phi$. Moreover, by Theorem $8.2^{\prime}$ of [1], the weak solutions of problem (P) are strong solutions. Thus, our goal is achieved if we show that condition (1) holds with this choice of $\Phi$ and $\Psi$. In fact, in this case, given a sequence $\left\{r_{n}\right\}_{n \in \mathbb{N}}$ of real numbers with $r_{n}>k$ and $r_{n} \rightarrow k$ we find a strong solution $u_{n}$ of problem (P) whose norm is smaller than $r_{n}$, so $\left\{u_{n}\right\}_{n \in \mathbb{N}}$ admits a subsequence, still denoted by $\left\{u_{n}\right\}_{n \in \mathbb{N}}$, weakly converging to $\bar{u}$ in $W_{0}^{1,2}(\Omega)$ and, by an embedding theorem, strongly converging to $\bar{u}$ in $L^{p}(\Omega)$. Now, given $v \in W_{0}^{1,2}(\Omega)$, taking into account the growth condition on $f$, we deduce from well known results that the sequence $\left\{f\left(\cdot, u_{n}(\cdot)\right) v(\cdot)\right\}_{n \in \mathbb{N}}$ converges almost everywhere to $f(\cdot \cdot \bar{u}(\cdot)) v(\cdot)$ and it is dominated by an $L^{1}(\Omega)$ function. Hence, $\int_{\Omega} f\left(x, u_{n}(x)\right) v(x) d x \rightarrow \int_{\Omega} f(x, \bar{u}(x)) v(x) d x$. By the previous considerations we conclude that $\bar{u}$ is a solution of problem $(\mathrm{P})$ with $\|\bar{u}\| \leq k$.

For all $r>0$ put

$$
\mu(r)=\sup _{\|v\| \leq r} \int_{\Omega}\left(\int_{0}^{v(x)} f(x, t) d t\right) d x .
$$

We observe that, thanks to condition (2) and the fact that $W_{0}^{1,2}(\Omega)$ is compactly embedded into $L^{q}(\Omega)$ for all $\left.q \in\right] 2,2 N /(N-2)[$, the function $\mu$ is well defined. Obviously, $\mu$ is non-decreasing in $] 0, \infty[$.

Now, as is easily seen, condition (1) is equivalent to

$$
\inf _{r>0} \inf _{s<r} \frac{\mu(r)-\mu(s)}{r^{2}-s^{2}}<\frac{1}{2} .
$$

This, in turn, is equivalent to the existence of $r>0$ and $s<r$ such that

$$
\mu(s)-\frac{1}{2} s^{2}>\mu(r)-\frac{1}{2} r^{2} .
$$

This holds if there exists $r>0$ such that

$$
\limsup _{h \rightarrow 0} \frac{\mu(r+h)-\mu(r)}{h}<r
$$

We show that this is indeed case. In fact, for every $r>0$ and every $h \in$ ]$-r, \infty[$ one has 


$$
\begin{aligned}
& \frac{1}{h}(\mu(r+h)-\mu(r)) \\
& \leq\left.\frac{1}{|h|}\right|_{\|v\| \leq r+h} \sup _{\Omega}\left(\int_{0}^{v(x)} f(x, t) d t\right) d x-\sup _{\|v\| \leq r} \int_{\Omega}\left(\int_{0}^{v(x)} f(x, t) d t\right) d x \mid \\
& =\frac{1}{|h|} \sup _{\|v\| \leq 1} \int_{\Omega}\left(\int_{0}^{(r+h) v(x)} f(x, t) d t\right) d x-\sup _{\|v\| \leq 1} \int_{\Omega}\left(\int_{0}^{r v(x)} f(x, t) d t\right) d x \mid \\
& \leq \frac{1}{|h|} \sup _{\|v\| \leq 1} \int_{\Omega} \int_{\|v\| \leq 1}^{(r+h) v(x)}|f(x, t)| d t \mid d x \\
& \leq \sup _{\Omega}\left(\alpha(x)\left|\frac{r+h-r}{h}\right| \cdot|v(x)|+\frac{\beta(x)}{q}\left|\frac{(r+h)^{q}-r^{q}}{h}\right| \cdot|v(x)|^{q}\right) d x \\
& \leq\|\alpha\|_{L^{s \gamma /(s \gamma-1)}(\Omega)}\|v\|_{L^{s \gamma}(\Omega)}+\frac{1}{q}\|\beta\|_{L^{1 /(1-\gamma)}(\Omega)}\|v\|_{L^{q / \gamma}(\Omega)}^{q}\left|\frac{(r+h)^{q}-r^{q}}{h}\right| \\
& \leq c_{1}(\gamma)\|\alpha\|_{L^{s \gamma /(s \gamma-1)}(\Omega)}+c_{2}(\gamma)^{q} \frac{1}{q}\|\beta\|_{L^{1 /(1-\gamma)}(\Omega)} \frac{(r+h)^{q}-r^{q}}{h} \mid,
\end{aligned}
$$

where $q=p+1$. Consequently, we have $\limsup _{h \rightarrow 0} \frac{1}{h}(\mu(r+h)-\mu(r)) \leq c_{1}(\gamma)\|\alpha\|_{L^{s \gamma /(s \gamma-1)}(\Omega)}+c_{2}(\gamma)^{q}\|\beta\|_{L^{1 /(1-\gamma)}(\Omega)} r^{p}$.

Thus, if we put

$$
\varphi(r)=c_{1}(\gamma)\|\alpha\|_{L^{s \gamma /(s \gamma-1)}(\Omega)}+c_{2}(\gamma)^{q}\|\beta\|_{L^{1 /(1-\gamma)}(\Omega)} r^{p}-r
$$

for all $r>0$, we only have to prove that $\inf _{r>0} \varphi(r)<0$. By using an elementary differential method, we see that the infimum is attained at

$$
r=\left(p c_{2}(\gamma)^{p+1}\|\beta\|_{L^{1 /(1-\gamma)}(\Omega)}\right)^{1 /(1-p)}
$$

and (2) entails that it is negative.

\section{References}

[1] S. Agmon, The $L_{p}$ approach to the Dirichlet problem, Ann. Scuola Norm. Sup. Pisa 13 (1959), 405-448.

[2] V. I. Burenkov and V. A. Gusakov, On precise constants in Sobolev imbedding theorems, Soviet Math. Dokl. 35 (1987), 651-655.

[3] D. Gilbarg and N. S. Trudinger, Elliptic Partial Differential Equations of Second Order, Springer, Berlin, 1977.

[4] A. S. Marano, Elliptic boundary value problems with discontinuous nonlinearities, Set-Valued Anal. 3 (1995), 167-180.

[5] B. Ricceri, A general variational principle and some of its applications, J. Comput. Appl. Math. 113 (2000), 401-410. 
[6] B. Ricceri, Infinitely many solutions of the Neumann problem for elliptic equations involving the p-Laplacian, Bull. London Math. Soc. 33 (2001), 331-340.

[7] -, On a classical existence theorem for nonlinear elliptic equations, in: Experimental, Constructive and Nonlinear Analysis, M. Théra (ed.), CMS Conf. Proc. 27, Canad. Math. Soc., 2000, 275-278.

[8] -, Existence and location of solutions to the Dirichlet problem for a class of nonlinear elliptic equations, Appl. Math. Lett. 14 (2001), 143-148.

[9] —, New results on local minima and their applications, in: Equilibrium Problems: Nonsmooth Optimization and Variational Inequality Models, F. Giannessi et al. (eds.), Kluwer, 2001, 255-268.

[10] C. L. Tang and X. P. Wu, Existence and multiplicity of solutions of semilinear elliptic equations, J. Math. Anal. Appl. 256 (2001), 1-12.

Department of Mathematics

University of Messina

98166 Sant'Agata - Messina, Italy

E-mail: Anello@dipmat.unime.it

Cordaro@dipmat.unime.it

Reçu par la Rédaction le 29.8.2002

Révisé le 18.11.2002 